\title{
Diagnostic accuracy of coronary computed tomography angiography for the evaluation of obstructive coronary artery disease in patients referred for transcatheter aortic valve implantation: a systematic review and meta-analysis
}

\author{
Marco Gatti ${ }^{1}$ (D) - Guglielmo Gallone ${ }^{2} \cdot$ Vittoria Poggi $^{1}$ - Francesco Bruno ${ }^{2} \cdot$ Alessandro Serafini $^{1}$. \\ Alessandro Depaoli ${ }^{1}$ • Ovidio De Filippo ${ }^{2} \cdot$ Federico Conrotto $^{2} \cdot$ Fatemeh Darvizeh $^{3} \cdot$ Riccardo Faletti $^{1}$. \\ Gaetano Maria De Ferrari $^{2}$ • Paolo Fonio ${ }^{1}$ • Fabrizio D'Ascenzo ${ }^{2}$
}

Received: 25 November 2021 / Revised: 2 January 2022 / Accepted: 19 January 2022 / Published online: 22 February 2022

(C) The Author(s) 2022

\begin{abstract}
Objective To evaluate the diagnostic accuracy of coronary computed tomography angiography (CCTA) for the evaluation of obstructive coronary artery disease (CAD) in patients referred for transcatheter aortic valve implantation (TAVI).

Methods EMBASE, PubMed/MEDLINE, and CENTRAL were searched for studies reporting accuracy of CCTA for the evaluation of obstructive CAD compared with invasive coronary angiography (ICA) as the reference standard. QUADAS-2 tool was used to assess the risk of bias. A bivariate random effects model was used to analyze, pool, and plot the diagnostic performance measurements across studies. Pooled sensitivity, specificity, positive ( + LR) and negative ( - LR) likelihood ratio, diagnostic odds ratio (DOR), and hierarchical summary ROC curve (HSROC) were evaluated. Prospero registration number: CRD42021252527. Results Fourteen studies (2533 patients) were included. In the intention-to-diagnose patient-level analysis, sensitivity and specificity for CCTA were 97\% (95\% CI: 94-98\%) and 68\% (95\% CI: 56-68\%), respectively, and + LR and -LR were 3.0 (95\% CI: 2.1-4.3) and 0.05 (95\% CI: $0.03-0.09$ ), with DOR equal to 60 (95\% CI: 30-121). The area under the HSROC curve was 0.96 (95\% CI: 0.94-0.98). No significant difference in sensitivity was found between single-heartbeat and other CT scanners (96\% (95\% CI: $90-99 \%)$ vs. 97\% (95\% CI: 94-98\%) respectively; $p=0.37)$, whereas the specificity of single-heartbeat scanners was higher ( $82 \%$ (95\% CI: 66-92\%) vs. 60\% (95\% CI: $46-72 \%$ ) respectively; $p<0.0001)$. Routine CCTA in the pre-TAVI workup could save $41 \%$ (95\% CI: $34-47 \%$ ) of ICAs if a disease prevalence of $40 \%$ is assumed.

Conclusions CCTA proved an excellent diagnostic accuracy for assessing obstructive CAD in patients referred for TAVI; the use of single-heartbeat CT scanners can further improve these findings.

Key Points

- CCTA proved to have an excellent diagnostic accuracy for assessing obstructive CAD in patients referred for TAVI.

- Routine CCTA in the pre-TAVI workup could save more than $40 \%$ of ICAs.

- Single-heartbeat CT scanners had higher specificity than others in the assessment of obstructive CAD in patients referred for TAVI.
\end{abstract}

Keywords Coronary artery disease $\cdot$ Transcatheter aortic valve replacement $\cdot$ Computed tomography angiography $\cdot$ Aortic valve stenosis

Marco Gatti

marcogatt17@gmail.com

Department of Surgical Sciences, Radiology Unit, University of Turin, Via Genova 3, 10126 Turin, Italy

2 Division of Cardiology, Department of Medical Science, University of Turin, Turin, Italy

3 School of Medicine, Vita-Salute San Raffaele University, 20121 Milan, Italy

$\begin{array}{ll}\text { Abbreviations } \\ \text { CAD } & \text { Coronary artery disease } \\ \text { CCTA } & \text { Coronary computed tomography angiography } \\ \text { CTA } & \text { Computed tomography angiography } \\ \text { DOR } & \text { Diagnostic odds ratio } \\ \text { HSROC } & \begin{array}{l}\text { Hierarchical summary receiver operating charac- } \\ \text { teristic curve }\end{array} \\ \text { ICA } & \text { Invasive coronary angiography } \\ \text {-LR } & \text { Negative likelihood ratio } \\ \text { + LR } & \text { Positive likelihood ratio }\end{array}$

\section{Abbreviation}

CCTA Coronary computed tomography angiography

CTA

giography

odds ratio 
PCI Percutaneous coronary intervention

PTP Post-test probability

TAVI Transcatheter aortic valve implantation

\section{Introduction}

Transcatheter aortic valve implantation (TAVI) represents the gold standard for treatment of severe aortic stenosis in patients at high and intermediate surgical risk [1]. Candidates for TAVI are an elderly and frail population with a high prevalence (up to $60 \%$ ) of coronary artery disease (CAD).

Current guidelines recommend pre-procedural screening for CAD before valvular intervention [1]. Computed tomography angiography (CTA) has become the standard imaging method for pre-procedural TAVI assessment because it can evaluate non-invasively both the aortic arch and the peripheral vessels $[1,2]$. Coronary computed tomography angiography (CCTA) has been recommended as an initial test in patients with low clinical likelihood of CAD due to its very high negative predictive value in this population [2], but the clinical value of this approach in the TAVI setting is still under evaluation. Indeed, patients with severe aortic stenosis have a high pre-test probability of obstructive CAD and present specific technical challenges to CCTA interpretation, including extensive coronary calcifications, frequent abnormal cardiac rhythm, and contraindication to nitrate administration [1].

To the best of our knowledge, only two meta-analyses [3, 4] have evaluated the diagnostic accuracy of CCTA in patients with aortic stenosis referred for aortic valve replacement (surgical or transcatheter). In the last few years, technological advances in CT scanners have resulted in improved image quality, allowing accurate assessment of coronary anatomy even in difficult technical settings [5, 6]; this aspect was not assessed by the two abovementioned meta-analyses, both published in 2018. Moreover, indications to TAVI are progressively extending to low-surgical-risk patients with lower pre-test probability of CAD $[7,8]$.

In this framework, the incorporation of coronary artery assessment into pre-TAVI CT evaluation has the potential to reduce the need for unnecessary ICA and total amount of contrast medium applied, making pre-procedural evaluation safer and faster with reduced cost $[9,10]$.

The aim of this systematic review and meta-analysis is to provide an updated overview of the diagnostic accuracy of CCTA for the evaluation of obstructive CAD among patients referred for TAVI.

\section{Materials and methods}

\section{Protocol and registration}

This systematic review and meta-analysis was performed according to the Preferred Reporting Items for Systematic Reviews and Meta-Analysis (PRISMA) extension for Diagnostic Test Accuracy (DTA) Studies [11]. The protocol was prospectively registered in the PROSPERO International register of systematic reviews with the ID number CRD42021252527.

\section{Eligibility criteria}

The primary aim of this study was to evaluate the diagnostic accuracy of CCTA for obstructive coronary stenosis among patients referred for TAVI. Studies reporting data on CCTA for the evaluation of obstructive $\mathrm{CAD}$ were deemed eligible if all the following inclusion criteria were respected: (1) CCTA performed with at least a 64-slice CT scanner; (2) ICA performed in all patients and used as the reference standard; (3) sensitivity and specificity were reported or assessed by the published data. Obstructive CAD was defined in the metaanalysis as a narrowing of the coronary lumen by more than $50 \%$ on CCTA and a lumen diameter reduction of more than $50 \%$ on ICA.

\section{Study endpoints}

The primary endpoint was the patient-level accuracy of CCTA to identify obstructive CAD. For the purpose of this analysis, non-evaluable segments were considered positive based on an intention to diagnose approach [12]. Secondary analyses included the evaluation of the accuracy of CCTA for obstructive $\mathrm{CAD}$ at the patient level, excluding patients with nonevaluable segments, at the vessel and at the segment level, evaluating also coronary artery bypass grafting $(\mathrm{CABG})$ and stented coronary artery segments. Sensitivity was analyzed according to the risk of bias and applicability. Subgroup analysis was performed based on CT scanner characteristics. We identified three main CT scanner subgroups: (1) whole-heart coverage CT scanner-scanners with extensive detector coverage on the z-axis (i.e., the $160-\mathrm{mm}$ scanners); (2) high temporal resolution CT scanners (i.e., dual-source scanners); (3) single-heartbeat CT scanners - scanner capable of acquiring the entire heart volume in a single beat, including both wholeheart CT scanners and high temporal resolution scanners with a large number of detectors (e.g., Somatom Force, Siemens Healthineers). In particular, we conducted three subgroup analyses to determine the effect of these technical CT parameters on diagnostic accuracy: (1) whole-heart coverage CT scanner vs. other CT scanners; (2) high temporal resolution 
CT scanners (i.e., dual-source CTs) vs. other CT scanners, and (3) single-heartbeat CT scanners vs. other CT scanners.

\section{Search strategy}

Excerpta Medica dataBASE (EMBASE), Medical Literature Analysis and Retrieval System Online (PubMed/MEDLINE), and Cochrane Central Register of Controlled Trials (CENTRAL) were searched up to May 1, 2021. The string used is reported in the Supplementary Material. The reference lists of selected articles were also searched manually to identify additional eligible studies.

\section{Data collection, data extraction, and risk of bias and applicability}

Two researchers (F.B. and A.S.) independently searched for studies fulfilling the inclusion criteria in a two-stage process: first by using title and abstract of the papers and then the full text. The reasons for excluding studies in this second phase were recorded. The results from both searches were compared and the discrepancies were discussed. In some cases of disagreement, the decision was reached by consultation with a third researcher (G.G.). All selected articles were automatically downloaded, imported, and de-duplicated in Microsoft Excel (Microsoft).

General characteristics included total number of patients, age, sex, body mass index, cardiovascular risk factor (i.e., diabetes, hypercholesterolemia, smoking, history, hypertension), known $\mathrm{CAD}$, previous percutaneous coronary intervention or CABG and atrial fibrillation. Moreover, main CT scanner characteristics were recorded: number of detector rows, dual-energy techniques, tube voltage tube current, contrast media concentration, contrast media volume, heart rate during acquisition, and mean dose-to-length product.

The quality assessment of diagnostic accuracy studies 2 (QUADAS-2) tool [13] was used to assess the risk of bias of included studies (reported in Supplementary Material).

\section{Statistical analysis}

Two-by-two contingency tables were extracted from each study and used to calculate sensitivity, specificity, positive $(+\mathrm{LR})$ and negative (-LR) likelihood ratio, and diagnostic odds ratio (DOR) with 95\% confidence interval (CI) of CCTA for the detection of significant coronary artery stenosis (in general, + LR $>10$ and $-\mathrm{LR}<0.1$ demonstrates a satisfactory diagnostic performance [14]). A bivariate random effects model was used to analyze, pool, and plot the diagnostic performance measurements across studies. Derived logit estimates of sensitivity, specificity, and respective variances were used to construct a hierarchical summary ROC curve (HSROC). Heterogeneity between studies was evaluated utilizing Cochran's $Q$ and Higgins $I^{2}$ statistics. Deeks' funnel plot was used to assess publication bias. The patient-level clinical accuracy of CCTA was evaluated using the likelihood ratios to calculate post-test probability based on Bayes' theorem with the use of Fagan's nomograms, Likelihood ratio scattergram, and probability modifying plot.

The analyses were performed with STATA (version 16.1, Stata Corp LP) using the MIDAS module [15] and MetaDTA (Diagnostic Test Accuracy Meta-Analysis v2.01) [16]. A $p$ value of less than 0.05 was considered statistically significant.

\section{Results}

\section{Literature search and study characteristics}

The PRISMA 2020 flow diagram [17] for systematic reviews is reported in Figure 1. Fourteen studies with a total of 2533 patients were included in the analysis. Tables 1 and 2 report the baseline characteristic of the included patients and of the CT scanner used.

\section{Assessment of study quality}

The QUADAS-2 Domain assessment is reported in Figure 2. The supplementary material contains the details of this analysis.

\section{Diagnostic accuracy: primary endpoint}

A total of 2228 patients were included in the analysis. For the purpose of this analysis, performed at the patient level, non-evaluable segments were considered positive based on an intention-to-diagnose approach. The pooled sensitivity and specificity for CCTA were $97 \%$ (94-98\%) and 68\% (56-68\%), respectively, and the + LR and -LR were 3.0 (2.1-4.3) and $0.05(0.03-0.09)$, with a DOR of 60 (30121). The HSROC had an AUC $=0.96(0.94-0.98)$. Table 3 shows sensitivity and specificity with \% (95\% CI) derived from each study included in the analysis. The summary forest plot and HSROC plot are reported in Figure 3.

The per-patient analysis revealed a + LR of 3.03 (2.12$4.33)$ and a -LR of $0.05(0.03-0.09)$ (i.e., with an estimated pre-test probability of CAD of $40 \%$, a positive CCTA could increase the post-test probability to $67 \%$ and a negative CCTA can decrease the post-test probability to $3 \%$, whereas in a hypothetical population with pre-test probability of $15 \%$, the post-test probability can reduce to less than $1 \%$ ).

Fagan's nomograms, with estimated pretest probability of $40 \%$ and $15 \%$, Likelihood ratio scattergram and probability modifying plot are reported in Figure 4. In summary, estimating a disease prevalence of $40 \%$ in a population of 1000 patients, the study of coronary arteries with CCTA before the 
Table 1 Baseline characteristics of the study population

\begin{tabular}{|c|c|c|c|c|c|c|c|c|c|c|c|c|c|c|}
\hline Author & $\begin{array}{l}\text { Publication date } \\
\text { Year }\end{array}$ & $\begin{array}{l}\mathrm{N} \\
\text { No. }\end{array}$ & $\begin{array}{l}\text { Age } \\
\text { Years }\end{array}$ & $\begin{array}{l}\text { Men } \\
\%\end{array}$ & $\begin{array}{l}\text { BMI } \\
\%\end{array}$ & $\begin{array}{l}\mathrm{DM} \\
\%\end{array}$ & $\begin{array}{l}\mathrm{HC} \\
\%\end{array}$ & $\begin{array}{l}\text { Smoke } \\
\%\end{array}$ & $\begin{array}{l}\mathrm{AF} \\
\%\end{array}$ & $\begin{array}{l}\text { Sinus rhythm } \\
\%\end{array}$ & $\begin{array}{l}\mathrm{HT} \\
\%\end{array}$ & $\begin{array}{l}\text { CAD } \\
\%\end{array}$ & $\begin{array}{l}\text { PCI } \\
\%\end{array}$ & $\begin{array}{l}\text { CABG } \\
\%\end{array}$ \\
\hline Pontone et al [26] & 2011 & 60 & 80 & 36.6 & 25 & 13 & 40 & 25 & 0 & 100 & 67 & 37 & 24 & 16 \\
\hline Andreini [30] & 2014 & 325 & 81.1 & 40.6 & 25.6 & 30 & 53 & 20 & 0 & 100 & 74 & 28 & 15 & 13 \\
\hline Hamdan et al [28] & 2015 & 115 & 81.4 & 43.5 & NR & 30 & 70 & 36.5 & 7.8 & 92.2 & 85 & 52.2 & 29 & 20 \\
\hline Harris et al [12] & 2015 & 100 & 79.6 & 61 & NR & 24 & 72 & 59 & 36 & 74 & 92 & 57 & 16 & 41 \\
\hline Opolsky et al [27] & 2015 & 475 & 82 & 41 & 27.5 & 32 & 48 & NR & 19 & 75 & 95 & 67 & 48 & 19 \\
\hline Matsumoto et al [25] & 2016 & 60 & 84.4 & 28.3 & 22.2 & NR & NR & NR & NR & NR & NR & 13 & 10 & 3.3 \\
\hline Rossi et al [19] & 2017 & 140 & 82.3 & 48.6 & 27.1 & 21 & 59 & 19 & 0 & 100 & 75 & 0 & 0 & 0 \\
\hline Annoni et al [22] & 2018 & 115 & 82.3 & 55.7 & 26.7 & 18 & 69 & 7 & 13 & 87 & 71 & 20.8 & 15 & 6.1 \\
\hline Hachulla [24] & 2019 & 84 & 84.65 & 48.1 & 26.9 & NR & NR & NR & NR & NR & NR & NR & NR & NR \\
\hline Strong et al [20] & 2019 & 200 & 83.4 & 40 & 26.6 & 28 & 74 & 21.5 & 34 & 76.5 & 93 & 0 & 0 & 0 \\
\hline Schicchi et al [29] & 2020 & 223 & 79.2 & NR & NR & $\mathrm{NR}$ & NR & NR & NR & NR & NR & 51.6 & 35 & 16.6 \\
\hline Gohmann et al [21] & 2020 & 388 & 79.6 & 50.8 & 29.2 & 13 & 59 & 8 & NR & 64.7 & 89 & 41.4 & 29 & 0 \\
\hline Shuai et al [23] & 2020 & 121 & 73.3 & 47.1 & 22.6 & 26 & 12 & 25 & 27 & 73.8 & 37 & NR & 0 & 0 \\
\hline Meier et al [18] & 2021 & 127 & 82.3 & 38.6 & 26.5 & 28 & 54 & NR & NR & NR & 77 & 38.6 & 17 & 0 \\
\hline
\end{tabular}

$N$ number, $B M I$ body mass index, $D M$ diabetes mellitus, $H C$ hypercholesterolemia, $A F$ atrial fibrillation, $H T$ hypertension, $C A D$ coronary artery disease, $P C I$ percutaneous coronary intervention, $C A B G$ coronary artery bypass graft, $N R$ not reported

TAVI procedure would correctly avoid 409 (95\% CI 335470) ICAs (Figure 5).

Table 4 provides a summary of the CCTA diagnostic performance for the evaluation of obstructive CAD among patients referred for TAVI at a patient, vessel, and segment level. The secondary analysis is reported in the Supplementary Material.

\section{Sub-analysis: sensitivity and subgroup analysis}

We found a high value for Cochran's $Q$ and $I^{2}$, which indicates the presence of heterogeneity in the studies. As a result, we visually assessed the forest plot and HSROC and a significant heterogeneity in specificity was found, particularly in the forest plot (Figure 4a), where some studies fell outside the combined $95 \%$ CI.

For the purpose of the sub-analysis, performed at the patient level, non-evaluable segments were considered positive based on an intention-to-diagnose approach.

A sensitivity analysis including only five studies [23, 26-29] without high or unclear risk of bias or concerns regarding applicability showed similar results to the analysis containing all studies: a total of 1003 patients were included, the pooled sensitivity and specificity for CCTA were $96 \%(92$ $-98 \%)$ and $79 \%(59-91 \%)$ respectively, and the + LR and -LR were $4.6(2.2-9.7)$ and $0.05(0.03-0.09)$, with a DOR of 94 (39-227). The HSROC had AUC $=0.97(0.95-0.98)$.

The results of the subgroup analysis based the various CT scanner features are summarized in Table 5 .
In summary, the use of a whole-heart coverage CT increased specificity $(p<0.001)$ but did not affect sensitivity $(p=0.26)$; the use of high temporal resolution scanners increased sensitivity $(p=0.02)$ but decreased specificity $(p<$ 0.001 ); and the use of single-heartbeat scanners increased specificity $(p<0.001)$ with no effect on sensitivity $(p=0.37)$.

To translate our findings into clinical practice, we estimated a disease prevalence of $40 \%$ in a 1000 -patient population and evaluated coronary arteries with different CT scanners: a whole-heart coverage CT scanner could correctly avoid 477 (95\% CI 340-552) ICAs, a high temporal resolution CT scanner could correctly avoid 357 (95\% CI 259-444) ICAs, and a single-heartbeat CT scanner could correctly avoid 494 (95\% CI 398-550) ICAs (Figure 6).

Furthermore, the percentage of non-evaluable patients with a whole-heart coverage CT scanner or a single-heartbeat CT scanner was $21.7 \%(13 / 60)$ compared to $37.1 \%(438 / 1180)$ with other CTs $(p=0.019)$. The percentage of non-evaluable patients using high temporal resolution CT scanners was $45.1 \%(368 / 815)$ compared to $19.5 \%$ (83/425) using other CTs $(p=0.001)$.

\section{Discussion}

In this systematic review and meta-analysis, we investigated the diagnostic accuracy of CCTA for the assessment of obstructive CAD among patients referred for TAVI. Overall, CCTA prior to TAVI procedure provides high sensitivity (97\%) with a good -LR (0.05). 


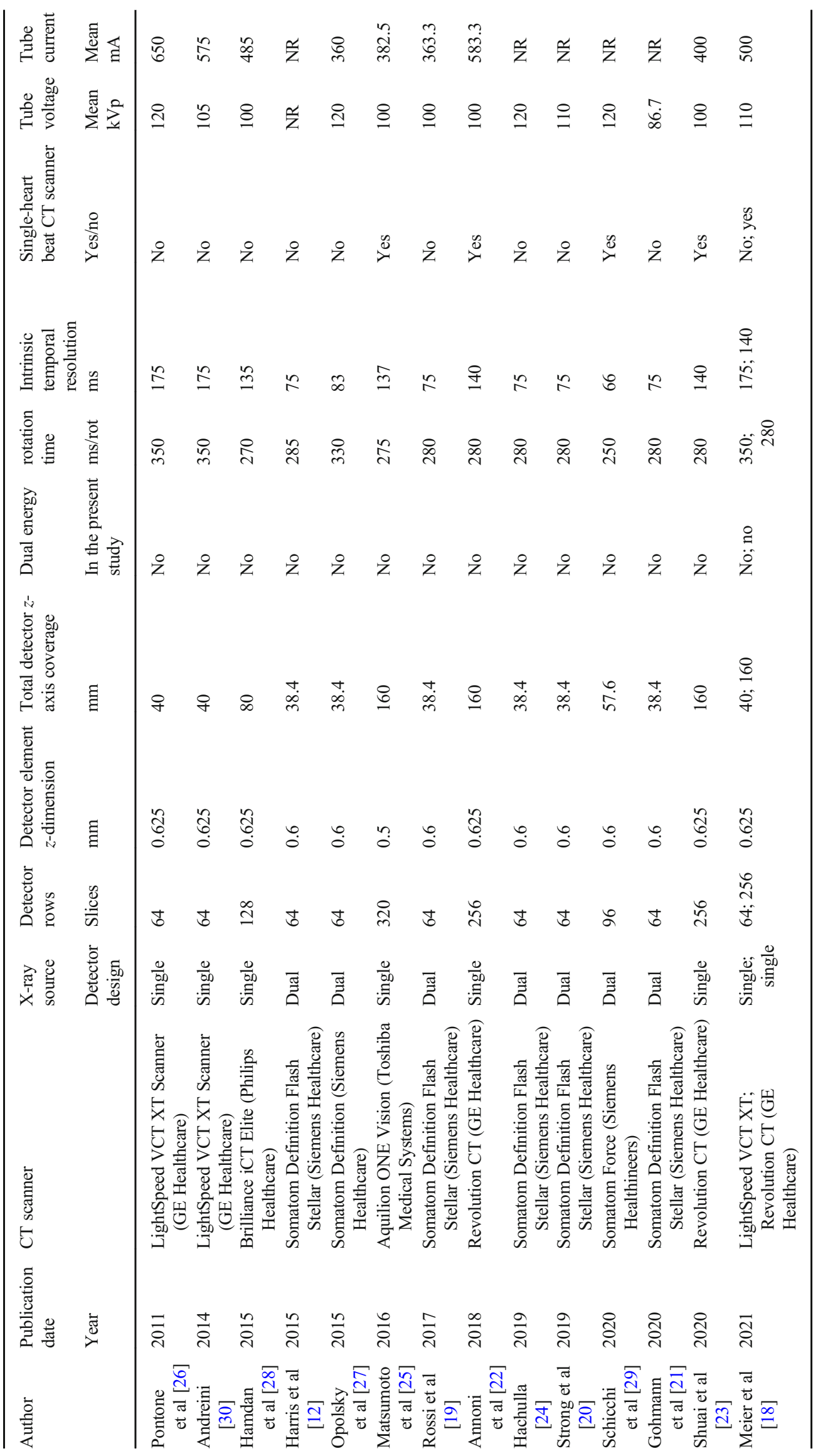


Fig. 1 Flow diagram

Identification of studies via databases and registers

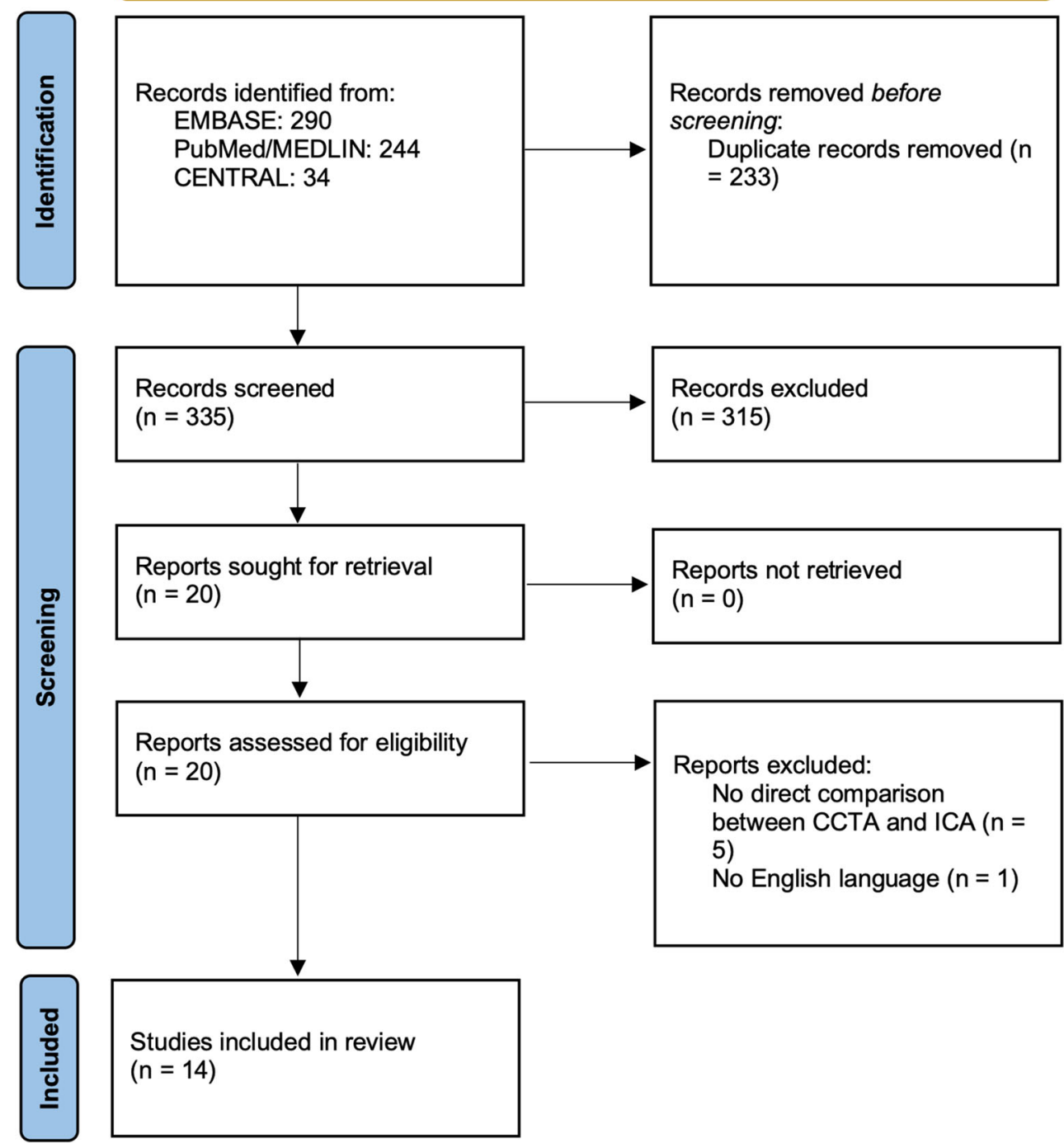

This result highlights the effectiveness of CCTA to rule out significant $\mathrm{CAD}$ and to reduce unnecessary ICA procedures by $40.9 \%$, aspiring its potential role as a gatekeeper test in this subgroup of patients. Furthermore, the use of new CT scanners, particularly single-heartbeat CT, has the potential to save the number of ICAs by up to $49.4 \%$.

A recent meta-analysis on the same topic by van den Boogert et al [3] with included seven studies on a total of 1275 patients (all of these studies/patients were also incorporated into our meta-analysis) reported sensitivity, specificity, positive predictive value, and negative predictive value of 95.3\% (93.3-96.9\%), 65.3\% (61.6-68.9\%), 70.8\% (68.6$72.9 \%)$, and $94.0 \%(91.6-95.8 \%)$ respectively. These results are very similar to our findings, despite the fact that the authors did not use a bivariate random effects model to summarize sensitivity, specificity, and their $95 \%$, but rather a fixed effects model. In addition, they did not estimate $+\mathrm{LR},-\mathrm{LR}$, and DOR.
Another meta-analysis on the performance of CCTA in patients with aortic stenosis undergoing surgery or transcatheter intervention by Chaikriangkrai et al [4] was performed. It included thirteen studies with a total of 1498 patients ( 6 of these studies, for a total of 1135 patients, were also incorporated into our meta-analysis); the results showed sensitivity $=95 \%(93-97 \%)$, specificity $=79 \%$ $(68-86 \%),+\mathrm{LR}=4.48(2.96-6.78),-\mathrm{LR}=0.06(0.04-$ $0.09)$ and $\mathrm{AUC}=0.96(0.95-0.98)$. The obtained specificity is slightly higher than our finding. This may be partially due to the population included in their study: in fact, in the subgroup analysis comparing patients who underwent surgery with those who underwent percutaneous intervention, the latter group showed a lower specificity (albeit not significant) $(83 \%(77-87 \%)$ vs. $74 \%$ (51-88\%)). This difference would be further increased by removing the study of Andreini et al [30] from their meta-analysis, thus increasing the specificity in the percutaneous intervention group 


\begin{tabular}{|c|c|c|c|c|c|c|c|}
\hline \multirow[t]{2}{*}{ Study } & \multicolumn{4}{|c|}{ RISK OF BIAS } & \multicolumn{3}{|c|}{ APPLICABILITY CONCERNS } \\
\hline & $\begin{array}{c}\text { PATIENT } \\
\text { SELECTION }\end{array}$ & $\begin{array}{c}\text { INDEX } \\
\text { TEST }\end{array}$ & $\begin{array}{c}\text { REFERENCE } \\
\text { STANDARD }\end{array}$ & $\begin{array}{c}\text { FLOW AND } \\
\text { TIMING }\end{array}$ & $\begin{array}{c}\text { PATIENT } \\
\text { SELECTION }\end{array}$ & $\begin{array}{c}\text { INDEX } \\
\text { TEST }\end{array}$ & $\begin{array}{c}\text { REFERENCE } \\
\text { STANDARD }\end{array}$ \\
\hline Pontone et al. & (2) & (:); & (-): & (2) & (2) & (-); & (-); \\
\hline Andreini et al. & (:) & (:) & (:) & (:) & (:) & (2) & ;) \\
\hline Hamdan et al. & ;): & (2): & (2) & (:) & (:) & (2): & (2) \\
\hline Harris et al. & (-) & (2): & $?$ & (2) & (:) & (2) & (-) \\
\hline Opolsky et al. & (2) & (:) & (:) & (:) & (:) & (:) & (2) \\
\hline Matsumoto et al. & (2) & (2): & (-) & (:) & (2) & (-): & (-) \\
\hline Rossi et al. & (-) & (:) & (-) & (2) & (2) & (-): & (-) \\
\hline Annoni et al. & (:) & (-): & $?$ & (:) & (-); & (-): & (-); \\
\hline Haculla et al. & (2): & (2): & (-): & $?$ & $?$ & (2): & (2) \\
\hline Strong et al. & (2): & (-); & (-) & (2) & (2) & (-) & (-); \\
\hline Schicchi et al. & (-): & (:) & ;) & (:) & (-) & (-): & (-) \\
\hline Gohmann et al. & (2) & (:) & (:) & (2) & (:) & (:) & (:) \\
\hline Shuai et al. & (-) & (2) & (:) & (2): & (;) & (-) & (-) \\
\hline Meier et al. & (-); & (2) & (2) & $?$ & (:) & (2) & (2) \\
\hline
\end{tabular}

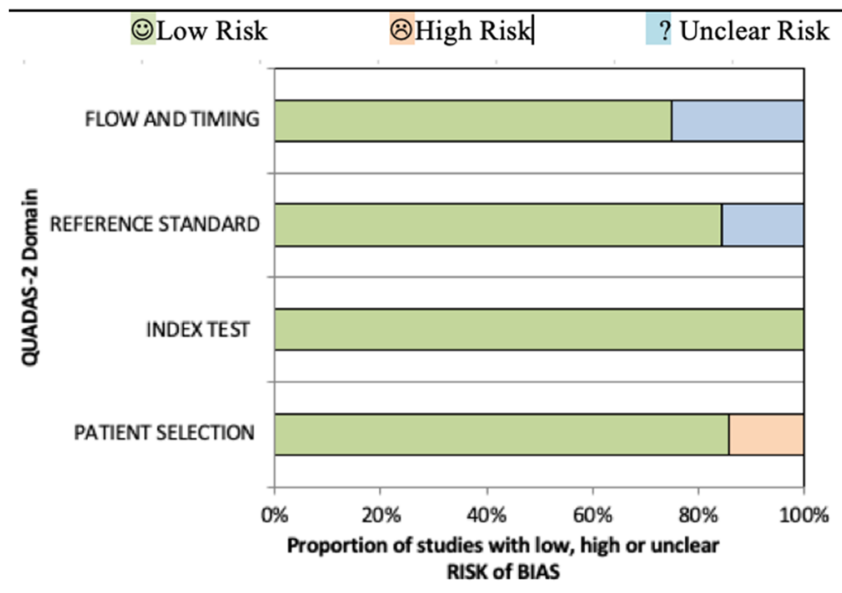

Fig.2 Quality assessment of diagnostic accuracy studies 2 (QUADAS-2) tool for risk of bias and applicability concern. Green represents low; yellow, high; and blue, unclear risk. On the top panel, QUADAS-2 was

even if it excluded non-evaluable patients. Another explanation could be the difference in disease prevalence between the two groups (48\% in transcatheter vs. $29 \%$ in surgical): subjects who underwent transcatheter replacement were typically more fragile and at higher risk than those who underwent surgery, and this may increase the number of nonevaluable segments, thus reducing specificity.

In accordance with the European Society of Cardiology (ESC) guidelines [1], CCTA with its high negative predictive value is considered useful to exclude $\mathrm{CAD}$ in patients undergoing TAVI who are at low risk for atherosclerosis. This is in perfect agreement with our findings: a patient with a pre-test probability of $15 \%$ has a post-test probability of less than $1 \%$, if the CCTA is negative.
口Low $\square$ High $\square$ Unclear

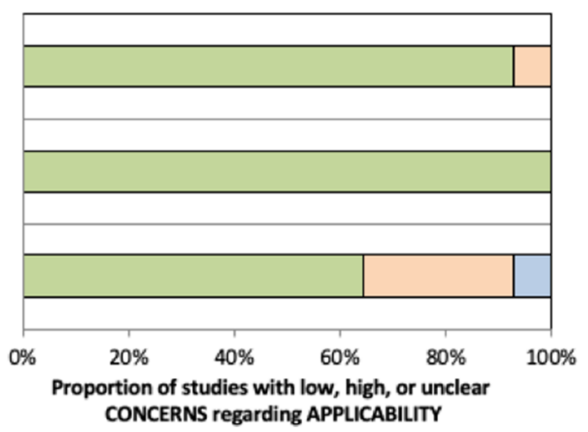

reported for each study and summarized in a bar graph on the bottom panel by stacked bars for each item

However, our data seem to support a slightly broader usefulness and feasibility of CCTA even for patients with intermediate risk of CAD. In fact, on the basis of our findings, a patient with a pre-test probability of $40 \%$ (i.e., the prevalence of the disease in our population $=40 \%$ ), in case of CCTA negativity, has a post-test probability of about $3 \%$. This last finding is supported by a recent meta-analysis [31] of prospective studies comparing CCTA with coronary angiography as the reference standard. It highlighted the role of CCTA in patients with a low-to-intermediate pretest probability of $\mathrm{CAD}$, emphasizing how this population could benefit the most from CCTA to rule in and rule out significant CAD.

The European Society of Cardiovascular Radiology (ESCR) consensus document [32] recently reported that 
Table 3 Summary sensitivities and specificities of CCTA for the identification of patients with obstructive coronary artery considering non-evaluable segments as positive

\begin{tabular}{|c|c|c|c|c|c|c|c|c|}
\hline Author & $\begin{array}{l}\text { Publication date } \\
\text { Year }\end{array}$ & $\begin{array}{l}\mathrm{N} \\
\text { No. }\end{array}$ & $\begin{array}{l}\text { TP } \\
\text { No. }\end{array}$ & $\begin{array}{l}\text { FN } \\
\text { No. }\end{array}$ & $\begin{array}{l}\text { FP } \\
\text { No. }\end{array}$ & $\begin{array}{l}\text { TN } \\
\text { No. }\end{array}$ & $\begin{array}{l}\mathrm{Se} \\
\%(\mathrm{CI} 95 \%)\end{array}$ & $\begin{array}{l}\mathrm{Sp} \\
\%(\mathrm{CI} 95 \%)\end{array}$ \\
\hline Pontone et al [26] & 2011 & 60 & 23 & 3 & 4 & 30 & $88.5(69.9-97.6)$ & $88.2(72.6-96.7)$ \\
\hline Hamdan et al [28] & 2015 & 115 & 47 & 2 & 18 & 48 & $95.9(86.0-99.5)$ & $72.7(60.4-83.0)$ \\
\hline Harris et al [12] & 2015 & 100 & 73 & 1 & 11 & 15 & $98.7(92.7-99.9)$ & $57.7(28.1-63.7)$ \\
\hline Opolsky et al [27] & 2015 & 475 & 265 & 5 & 129 & 76 & $98.2(95.7-99.4)$ & $37.1(30.5-44.1)$ \\
\hline Matsumoto et al [25] & 2016 & 66 & 22 & 2 & 21 & 21 & $91.7(73.0-99.0)$ & $50.0(34.2-65.8)$ \\
\hline Rossi et al [19] & 2017 & 145 & 58 & 5 & 37 & 45 & $92.1(82.4-97.4)$ & $54.9(43.5-65.9)$ \\
\hline Annoni et al [22] & 2018 & 115 & 22 & 1 & 12 & 80 & $95.7(78.1-99.9)$ & $87.0(78.3-93.1)$ \\
\hline Strong et al [20] & 2019 & 200 & 69 & 0 & 76 & 55 & $100.0(94.8-100.0)$ & $42.0(33.4-50.9)$ \\
\hline Schicchi et al [29] & 2020 & 223 & 44 & 1 & 20 & 158 & $97.8(88.2-99.9)$ & $88.8(61.9-82.9)$ \\
\hline Gohmann et al [21] & 2020 & 388 & 135 & 3 & 137 & 113 & $97.8(93.8-99.6)$ & $45.2(32.9-51.6)$ \\
\hline Shuai et al [23] & 2020 & 130 & 28 & 1 & 11 & 90 & $96.6(82.2-99.9)$ & $89.1(81.4-94.4)$ \\
\hline Meier et al [18] & 2021 & 127 & 43 & 6 & 33 & 45 & $87.8(75.2-95.4)$ & $57.7(46.0-68.8)$ \\
\hline
\end{tabular}

$N$ number of patients, $T P$ true positive, $F P$ false positive, $F N$ false negative, $T N$ true negative, $S e$ sensitivity, $S p$ specificity

CCTA should not be used routinely for pre-procedural assessment of CAD; however, they also added that, as technology evolves, CCTA can be used on a "case-by-case" basis, according to local expertise and available equipment and primarily to exclude significant coronary stenosis. This last statement was one of the starting points for our subgroup analyses. Technological advances in CT scanners were notable in recent years: the ideal CT scanner for cardiac imaging has high spatial and temporal resolution, covers the entire cardiac volume in a single rotation, and is ALARP (as low as reasonably practicable) compliant [33].
The abovementioned meta-analyses on a similar population [3, 4], published in 2018, did not analyze this aspect: (a) Regarding the high temporal resolution CT scanners, only three studies [12, 19, 27] analyzing 100, 475, and 145 patients, respectively, were included. (b) Concerning whole heart and single-heartbeat CT scanner, only one study [25] containing 66 patients was included.

In the period between those meta-analyses and our study, seven more papers using these technologies were published [18, 20-24, 29]. Therefore, we planned a sub-group analysis to explore the benefits of high intrinsic TR obtained by dual-
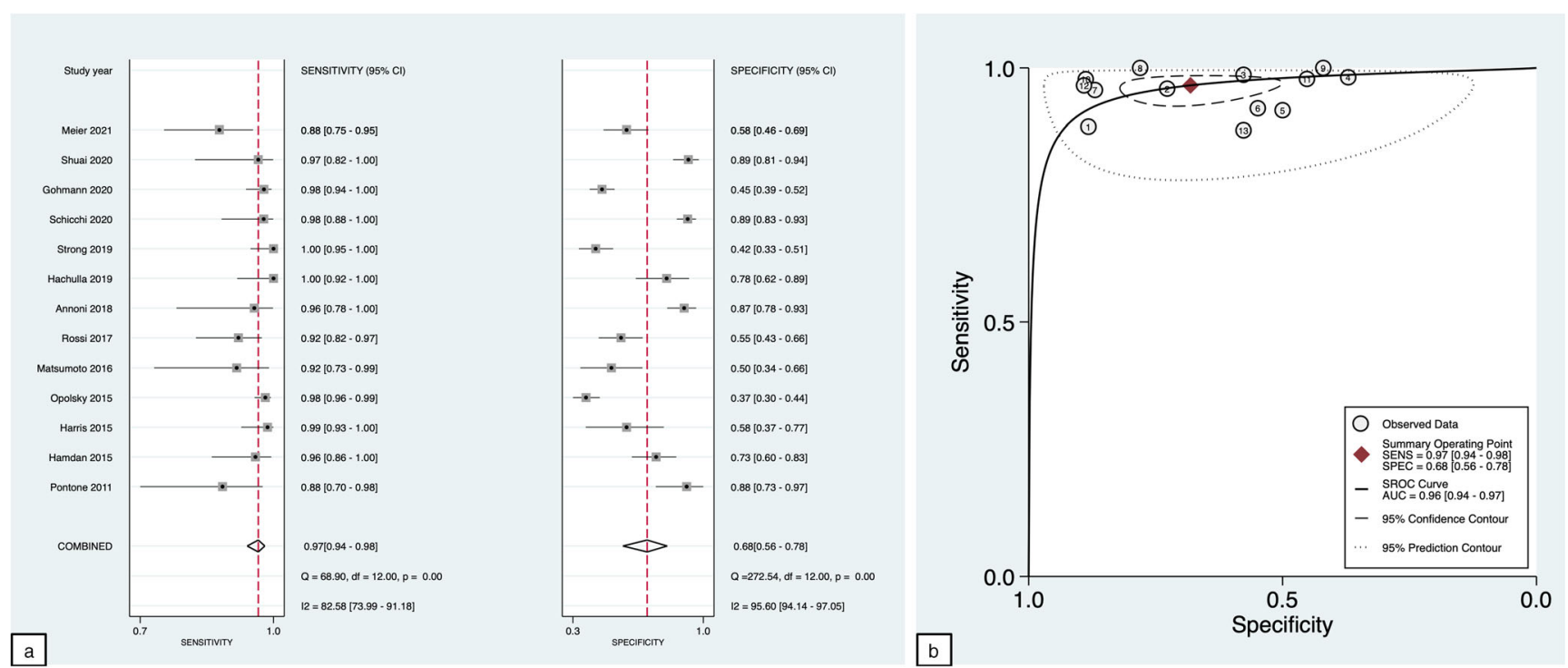

Fig. 3 Summary forest plot is shown as paired plots, with sensitivity and specificity paired (a). HSROC plot at a patient-based level considering positive the nonvaluable segments with confidence and prediction regions around mean operating sensitivity and specificity point (b) 

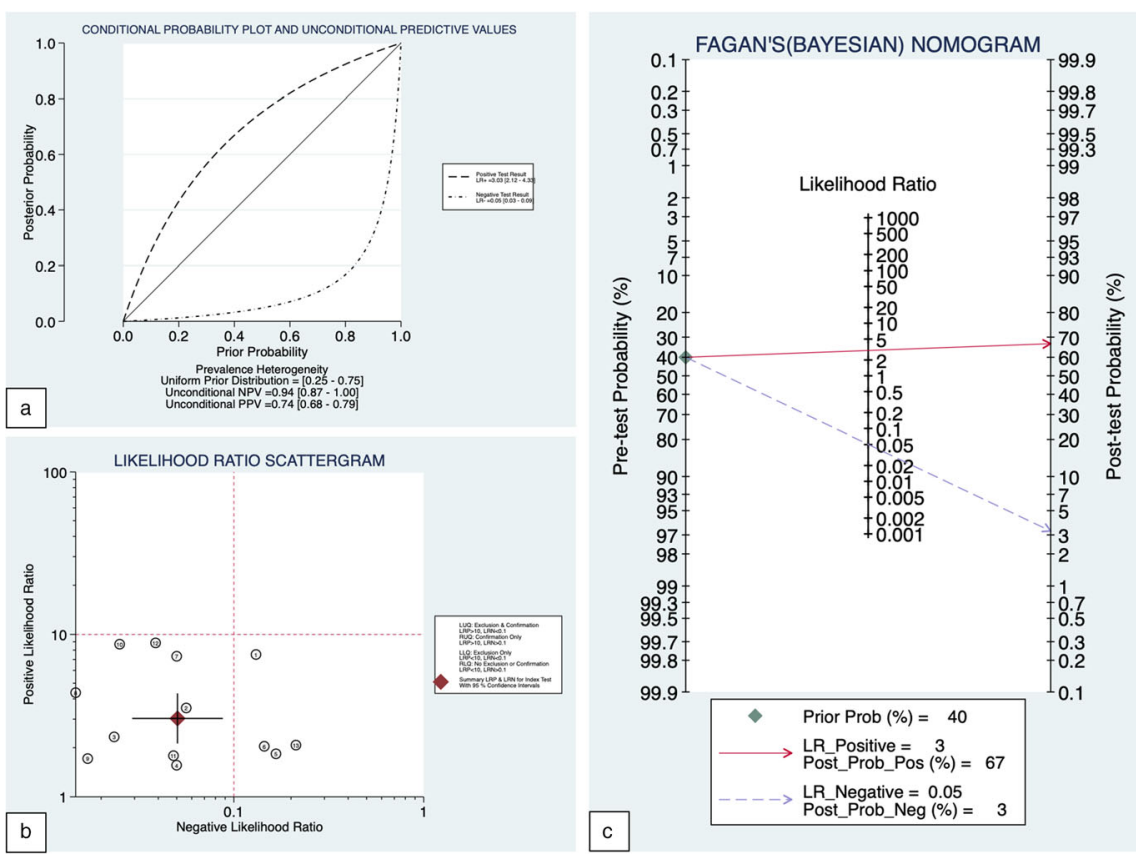

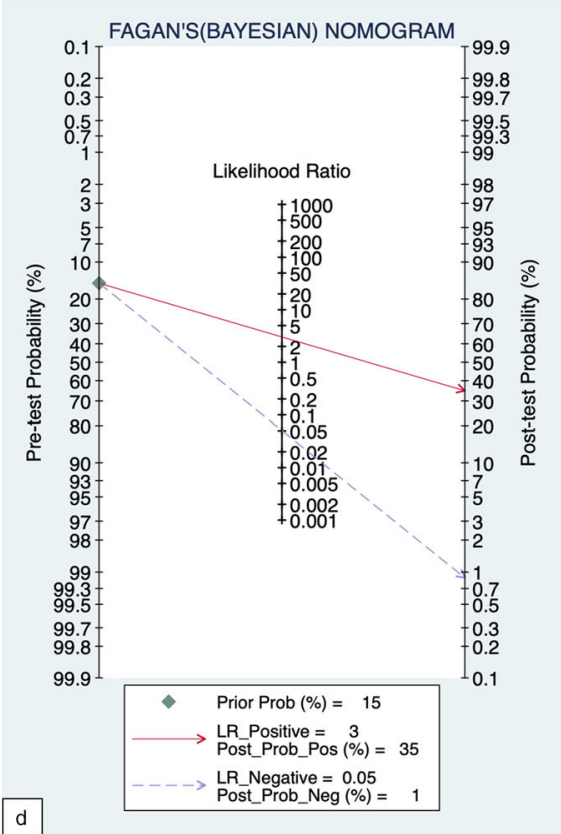

Fig. 4 a The conditional probability modifying plot is a graphical sensitivity analysis of predictive value along a prevalence continuum designating low-risk to high-risk populations. It shows distinct curves for positive and negative testing. The user draws a vertical line from the chosen pre-test probability to the appropriate likelihood ratio line, then reads the post-test probability from the vertical scale. b The likelihood ratio scattergram represents the summary point of likelihood ratios calculated as functions of mean sensitivity and specificity. The summary point is located in the left lower quadrant: the CCTA has a likelihood ratio positive $<10$ and a likelihood ratio negative $<0.1$. Based on these considerations, the CCTA in patients referred for TAVI is useful for exclusion of CAD (when negative) rather than confirmation (when positive). c, d Fagan's nomograms, with estimated pretest probability of $40 \%$ and $15 \%$, respectively. A Fagan plot has a vertical axis on the left with the prior log-odds, a vertical axis in the middle with the log-likelihood ratio, and a vertical axis on the right with the posterior log-odds. The lines are then traced from the prior probability on the left to the likelihood ratios in the center, and then to the posterior probabilities on the right. Both plots highlight the strength of the CCTA in excluding the presence of CAD, with residual post-test probabilities of $3 \%$ and $1 \%$, respectively source CT scanner, whole-heart coverage CT scanner, and single-heartbeat CT scanner. According to our findings, the use of CT scanners with high temporal resolution seems to improve sensitivity with lower specificity, whereas the use of whole-heart coverage and single-heartbeat $\mathrm{CT}$ scanners resulted in a higher specificity.

Single-heartbeat CT scanners in the evaluation of obstructive CAD prior to TAVI could correctly avoid up to $49.4 \%$ of ICAs. This data may be at least partially related to the increased number of segments, which allows accurate assessment of coronary arteries even in challenging population. The nonassessable segments were considered positive, leading to higher sensitivity and lower specificity in case of a high number of non-assessable segments. Our results confirm in a larger population the findings of Meier et al [18], who reported that the use of a 64-row scanner (compared to a 256-row scanner) for evaluating $\mathrm{CAD}$ in a pre-TAVI population was the only parameter in a multivariate analysis to be associated with a high risk of unanalyzable images. Our findings are consistent also with the meta-analysis of Haase et al [31], who reported that using a CT scanner with more than 64 detector rows led to higher sensitivity $(93.4 \%$ vs $86.5 \%, p=0.002)$ and specificity $(84.4 \%$ vs $72.6 \%, p=0.001$ ) in ruling out or confirming CAD patients with a pretest probability of CAD ranging from 7 to $67 \%$.

From a clinical point of view, it has been reported that patients with a large area of myocardium at ischemic risk
Fig. 5 CCTA's estimated impact in 1000 patients referred for TAVI. The numbers in brackets represent $95 \%$ confidence intervals

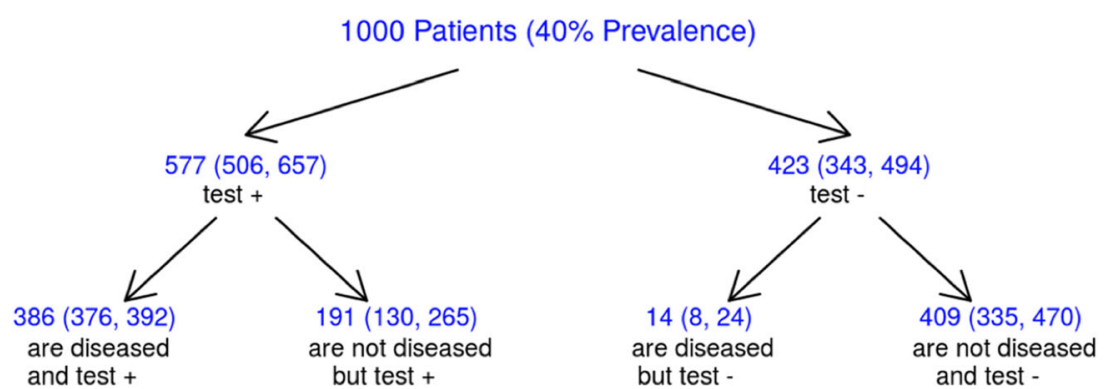


Table 4 CCTA diagnostic performance for the evaluation of obstructive coronary artery disease in patients referred for TAVI

\begin{tabular}{|c|c|c|c|c|c|c|c|c|c|c|}
\hline Analy & $\begin{array}{l}N \\
\text { No. }\end{array}$ & $\begin{array}{l}\text { TP } \\
\text { No. }\end{array}$ & $\begin{array}{l}\text { FP } \\
\text { No. }\end{array}$ & $\begin{array}{l}\text { FN } \\
\text { No. }\end{array}$ & $\begin{array}{l}\text { TN } \\
\text { No. }\end{array}$ & $\begin{array}{l}\mathrm{Se} \\
\%(\mathrm{CI} 95 \%)\end{array}$ & $\begin{array}{l}\mathrm{Sp} \\
\%(\mathrm{CI} 95 \%)\end{array}$ & $\begin{array}{l}+\mathrm{LR} \\
\%(\mathrm{CI} 95 \%)\end{array}$ & $\begin{array}{l}\text { - LR } \\
\%(\text { CI 95\%) }\end{array}$ & $\begin{array}{l}\text { DOR } \\
n(\text { CI 95\%) }\end{array}$ \\
\hline atient level 1a) & 2228 & 872 & 518 & 30 & 808 & 97( & $68(5$ & 3.0 & 0.05 & 60 \\
\hline Patient level 1b) & 794 & 252 & 123 & 16 & 403 & $94(89-97)$ & $80(64-90)$ & $4.6(2.4-8.8)$ & $0.08(0.04-0.14)$ & $59(23-149)$ \\
\hline Vessel level & 6865 & 1307 & 1529 & 102 & 3927 & 92 & 79 & $6.3)$ & $0.10(0.07-$ & $42(25-74)$ \\
\hline Segment level & 13525 & 1379 & 1408 & 81 & 10657 & $95(89-98)$ & $91(83-95)$ & $10.6(5.6-20.4)$ & $0.06(0.03-0.12)$ & $189(61-583)$ \\
\hline
\end{tabular}

1a) Considering as positive the nonvaluable segments; 1b) including only patients with all segments evaluable

$N$ number of cases included, $T P$ true positive, $F P$ false positive, $F N$ false negative, $T N$ true negative, $S e$ sensitivity, $S p$ specificity, $+L R$ positive likelihood ratio, $-L R$ negative likelihood ratio, DOR diagnostic odds ratio

Table 5 CCTA diagnostic performance (comparison between single-heartbeat CT scanner vs. others) for the evaluation of obstructive coronary artery disease in patients referred for TAVI

\begin{tabular}{|c|c|c|c|c|c|c|c|c|c|c|c|c|}
\hline CT scanner feature & Present & $\begin{array}{l}N \\
\text { No. }\end{array}$ & $\begin{array}{l}\text { TP } \\
\text { No. }\end{array}$ & $\begin{array}{l}\text { FP } \\
\text { No. }\end{array}$ & $\begin{array}{l}\mathrm{FN} \\
\text { No. }\end{array}$ & $\begin{array}{l}\mathrm{TN} \\
\text { No. }\end{array}$ & $\begin{array}{l}\mathrm{Se} \\
\%(\mathrm{CI} \\
95 \%)\end{array}$ & $\begin{array}{l}\mathrm{Sp} \\
\%(\mathrm{CI} \\
95 \%)\end{array}$ & $\begin{array}{l}+\mathrm{LR} \\
\%(\mathrm{CI} 95 \%)\end{array}$ & $\begin{array}{l}-\mathrm{LR} \\
\%(\mathrm{CI} 95 \%)\end{array}$ & $\begin{array}{l}\text { DOR } \\
n(\text { CI } 95 \%)\end{array}$ & $\begin{array}{l}\text { HSROC } \\
\text { AUC }\end{array}$ \\
\hline \multirow[t]{2}{*}{$\begin{array}{l}\text { Whole-heart coverage } \\
\text { CT scanner }\end{array}$} & Yes & 311 & 72 & 44 & 4 & 191 & $\begin{array}{c}95(86- \\
98)\end{array}$ & $\begin{array}{c}80(57- \\
92)\end{array}$ & $\begin{array}{c}4.6(1.9- \\
11.2)\end{array}$ & $\begin{array}{c}0.06(0.02- \\
0.20)\end{array}$ & $73(12-454)$ & $\begin{array}{l}0.96(0.94- \\
0.97)\end{array}$ \\
\hline & No & 1790 & 757 & 441 & 20 & 572 & $\begin{array}{c}97(95- \\
98)\end{array}$ & $\begin{array}{c}65(50- \\
77)\end{array}$ & $2.8(1.9-4.1)$ & $\begin{array}{c}0.04(0.02- \\
0.08)\end{array}$ & $64(31-130)$ & $\begin{array}{c}0.97(0.95- \\
0.98)\end{array}$ \\
\hline \multirow{2}{*}{$\begin{array}{l}\text { High temporal } \\
\text { resolution } \\
\text { CT scanners }\end{array}$} & Yes & 1615 & 687 & 419 & 15 & 494 & $\begin{array}{c}98(96- \\
99)\end{array}$ & $\begin{array}{c}59(43- \\
74)\end{array}$ & $2.4(1.6-3.6)$ & $\begin{array}{c}0.03(0.01- \\
0.08)\end{array}$ & $73(26-206)$ & $\begin{array}{l}0.97(0.95- \\
0.98)\end{array}$ \\
\hline & No & 486 & 142 & 66 & 9 & 269 & $\begin{array}{c}94(89- \\
97)\end{array}$ & $\begin{array}{c}80(66- \\
89)\end{array}$ & $4.7(2.6-8.5)$ & $\begin{array}{c}0.07(0.04- \\
0.14)\end{array}$ & $64(23-179)$ & $\begin{array}{c}0.95(0.92- \\
0.96)\end{array}$ \\
\hline \multirow[t]{2}{*}{$\begin{array}{l}\text { Single-heartbeat } \\
\text { CT scanner }\end{array}$} & Yes & 1567 & 713 & 421 & 19 & 414 & $\begin{array}{c}96(90- \\
99)\end{array}$ & $\begin{array}{c}82(66- \\
92)\end{array}$ & $\begin{array}{c}5.4(2.6- \\
11.3)\end{array}$ & $\begin{array}{c}0.05(0.02- \\
0.14)\end{array}$ & $\begin{array}{c}112(23- \\
548)\end{array}$ & $\begin{array}{l}0.97(0.95- \\
0.98)\end{array}$ \\
\hline & No & 534 & 116 & 64 & 5 & 349 & $\begin{array}{c}97(94- \\
98)\end{array}$ & $\begin{array}{c}60(46- \\
72)\end{array}$ & $2.4(1.7-3.3)$ & $\begin{array}{c}0.05(0.03- \\
0.09)\end{array}$ & $47(25-91)$ & $\begin{array}{l}0.95(0.93- \\
0.97)\end{array}$ \\
\hline
\end{tabular}

$N$ number of cases included, $T P$ true positive, $F P$ false positive, $F N$ false negative, $T N$ true negative, $S e$ sensitivity, $S p$ specificity, $+L R$ positive likelihood ratio, $-L R$ negative likelihood ratio, DOR diagnostic odds ratio

may benefit the most from revascularization intervention prior to TAVI, and the proximal segment stenosis is prognostically more important than distal [34]. Unfortunately, the only study included in this meta-analysis that allows this type of analysis is that of Andreini et al [30], where the exclusion of nonassessable segments gives a boost to the specificity of CCTA, which appears superior even in the study of distal vs. proximal segments $[35,36]$.

Further studies are needed to evaluate the real impact of CCTA in clinical practice; a randomized controlled trial could be proposed to evaluate the prognostic impact of patients undergoing CCTA pre-TAVI vs. a group not undergoing CCTA; also, the actual potential of CCTA in the study of proximal segments in such a selected population remains to be explored.

This meta-analysis has some limitations. First of all, a relatively low number of studies met the selection criteria. Unfortunately, only a fraction of the studies reported the analyses at patient level, either by considering nonanalyzable segments as positive or by excluding them,
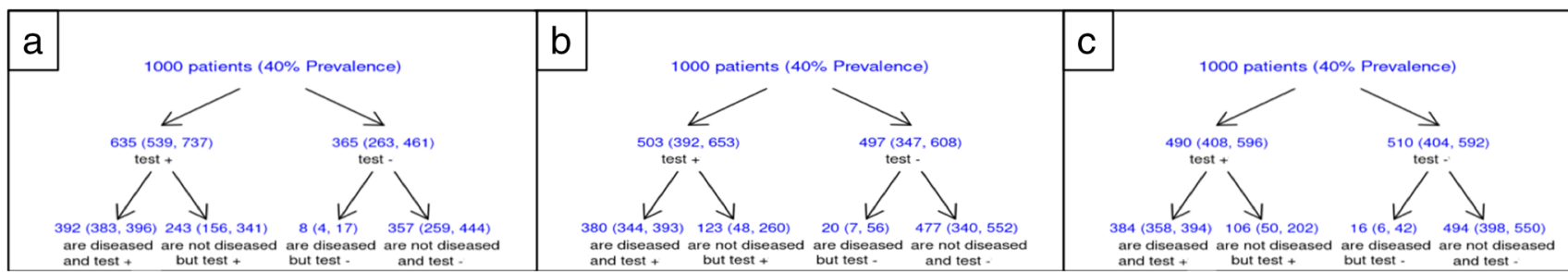

Fig. 6 Impact of high temporal resolution CT scanners (i.e., dual-source CTs) (a), whole-heart coverage CT scanner (b), and single-heartbeat CT scanner (c) in 1000 patients referred for TAVI. The numbers in brackets represent $95 \%$ confidence intervals 
and therefore it was not possible to create $3 \times 2$ tables [37]. Moreover, despite relevance from a clinical point of view, we were not able to compare performance among high vs. low calcium and arrhythmic and vs. nonarrhythmic patients. Almost all studies included are retrospective cohorts; only one is prospective and none a randomized controlled study. In addition, many of the included studies are small in size. The studies' overall quality was however adequate for analysis according to the QUADAS-2 evaluation, despite the presence of some unclear or high-risk items.

In conclusion, CCTA proved to have excellent diagnostic accuracy for assessing obstructive CAD in patients referred for TAVI. Routine CCTA assessment of coronary arteries as part of the pre-TAVI workup could save more than $40 \%$ of ICAs. The use of single-heartbeat CT scanners, which provide higher specificity, can further improve these findings.

Supplementary Information The online version contains supplementary material available at https://doi.org/10.1007/s00330-022-08603-y.

Funding The authors state that this work has not received any funding.

\section{Declarations}

Guarantor The scientific guarantor of this publication is Prof. Paolo Fonio, chief of Diagnostic and Interventional Radiology at the University of Turin.

Conflict of interest The authors of this manuscript declare no relationships with any companies whose products or services may be related to the subject matter of the article.

Statistics and biometry Dott. Marco Gatti, one of the authors, kindly provided statistical advice for this manuscript.

Informed consent The protocol was prospectively registered in the PROSPERO International register of systematic reviews with the ID number CRD42021252527.

Ethical approval Institutional Review Board approval was obtained.

\section{Methodology \\ - Meta-analysis}

Open Access This article is licensed under a Creative Commons Attribution 4.0 International License, which permits use, sharing, adaptation, distribution and reproduction in any medium or format, as long as you give appropriate credit to the original author(s) and the source, provide a link to the Creative Commons licence, and indicate if changes were made. The images or other third party material in this article are included in the article's Creative Commons licence, unless indicated otherwise in a credit line to the material. If material is not included in the article's Creative Commons licence and your intended use is not permitted by statutory regulation or exceeds the permitted use, you will need to obtain permission directly from the copyright holder. To view a copy of this licence, visit http://creativecommons.org/licenses/by/4.0/.

\section{References}

1. Baumgartner H, Falk V, Bax JJ et al (2017) 2017 ESC/EACTS Guidelines for the management of valvular heart disease. Eur Heart J 38:2739-2791. https://doi.org/10.1093/eurheartj/ehx391

2. Knuuti J, Wijns W, Saraste A et al (2020) 2019 ESC Guidelines for the diagnosis and management of chronic coronary syndromes. Eur Heart J 41:407-477. https://doi.org/10.1093/eurheartj/ehz425

3. van den Boogert TPW, Vendrik J, Claessen BEPM et al (2018) CTCA for detection of significant coronary artery disease in routine TAVI work-up : a systematic review and meta-analysis. Neth Heart J 26:591-599. https://doi.org/10.1007/s12471-018-1149-6

4. Chaikriangkrai K, Jhun HY, Shantha GPS et al (2018) Diagnostic accuracy of coronary computed tomography before aortic valve replacement: systematic review and meta-analysis. J Thorac Imaging 33:207-216. https://doi.org/10.1097/RTI. 0000000000000322

5. Andreini D, Mushtaq S, Pontone G et al (2018) Diagnostic performance of coronary CT angiography carried out with a novel wholeheart coverage high-definition CT scanner in patients with high heart rate. Int J Cardiol 257:325-331. https://doi.org/10.1016/j. ijcard.2017.10.084

6. Kwan AC, Gransar H, Tzolos E et al (2021) The accuracy of coronary CT angiography in patients with coronary calcium score above 1000 Agatston Units: comparison with quantitative coronary angiography. J Cardiovasc Comput Tomogr. https://doi.org/10. 1016/j.jcct.2021.03.007

7. Mack MJ, Leon MB, Thourani VH et al (2019) Transcatheter aortic-valve replacement with a balloon-expandable valve in lowrisk patients. N Engl J Med 380:1695-1705. https://doi.org/10. 1056/NEJMoa1814052

8. Leon MB, Smith CR, Mack MJ et al (2016) Transcatheter or surgical aortic-valve replacement in intermediate-risk patients. N Engl J Med 374:1609-1620. https://doi.org/10.1056/NEJMoa1514616

9. Usman MS, Rawasia WF, Siddiqi TJ et al (2019) Meta-analysis evaluating the safety and efficacy of transcarotid transcatheter aortic valve implantation. Am J Cardiol 124:1940-1946. https://doi.org/ 10.1016/j.amjcard.2019.09.015

10. Li Y-M, Mei F-Y, Yao Y-J et al (2021) Causes and predictors of readmission after transcatheter aortic valve implantation : a metaanalysis and systematic review. Herz 46:1-8. https://doi.org/10. 1007/s00059-019-04870-6

11. McInnes MDF, Moher D, Thombs BD et al (2018) Preferred Reporting Items for a Systematic Review and Meta-analysis of Diagnostic Test Accuracy Studies: the PRISMA-DTA statement. JAMA 319:388-396. https://doi.org/10.1001/jama.2017.19163

12. Harris BS, De Cecco CN, Schoepf UJ et al (2015) Dual-source CT imaging to plan transcatheter aortic valve replacement: accuracy for diagnosis of obstructive coronary artery disease. Radiology 275 : 80-88. https://doi.org/10.1148/radiol.14140763

13. Whiting PF, Rutjes AWS, Westwood ME et al (2011) QUADAS-2: a revised tool for the quality assessment of diagnostic accuracy studies. Ann Intern Med 155:529-536. https://doi.org/10.7326/ 0003-4819-155-8-201110180-00009

14. Devillé WL, Buntinx F, Bouter LM et al (2002) Conducting systematic reviews of diagnostic studies: didactic guidelines. BMC Med Res Methodol 2:9. https://doi.org/10.1186/1471-2288-2-9

15. Dwamena B (2007) MIDAS: Stata module for meta-analytical integration of diagnostic test accuracy studies. Statistical Software Components S456880, Boston College Department of Economics. Revised 05 Feb 2009

16. Patel A, Cooper N, Freeman S, Sutton A (2021) Graphical enhancements to summary receiver operating characteristic plots to facilitate the analysis and reporting of meta-analysis of diagnostic test 
accuracy data. Res Synth Methods 12:34-44. https://doi.org/10. 1002/jrsm. 1439

17. Page MJ, McKenzie JE, Bossuyt PM et al (2021) The PRISMA 2020 statement: an updated guideline for reporting systematic reviews. BMJ 372:n71. https://doi.org/10.1136/bmj.n71

18. Meier D, Depierre A, Topolsky A et al (2021) Computed tomography angiography for the diagnosis of coronary artery disease among patients undergoing transcatheter aortic valve implantation. J Cardiovasc Transl Res. https://doi.org/10.1007/s12265-02110099-8

19. Rossi A, De Cecco CN, Kennon SRO et al (2017) CT angiography to evaluate coronary artery disease and revascularization requirement before trans-catheter aortic valve replacement. J Cardiovasc Comput Tomogr 11:338-346. https://doi.org/10.1016/j.jcct.2017. 06.001

20. Strong C, Ferreira A, Teles RC et al (2019) Diagnostic accuracy of computed tomography angiography for the exclusion of coronary artery disease in candidates for transcatheter aortic valve implantation. Sci Rep 9:19942. https://doi.org/10.1038/s41598-019-56519-3

21. Gohmann RF, Lauten P, Seitz P et al (2020) Combined coronary CT-angiography and TAVI-planning: a contrast-neutral routine approach for ruling-out significant coronary artery disease. J Clin Med 9:1623. https://doi.org/10.3390/jcm9061623

22. Annoni AD, Andreini D, Pontone G et al (2018) CT angiography prior to TAVI procedure using third-generation scanner with wide volume coverage: feasibility, renal safety and diagnostic accuracy for coronary tree. Br J Radiol 91:20180196. https://doi.org/10. 1259/bjr.20180196

23. Shuai T, Li W, You Y et al (2020) Combined coronary CT angiography and evaluation of access vessels for TAVR patients in freebreathing with single contrast medium injection using a $16-\mathrm{cm}-$ wide detector CT. Acad Radiol 28(12):1662-1668. https://doi.org/ 10.1016/j.acra.2020.08.003

24. Hachulla A-L, Noble S, Ronot M et al (2019) Low iodine contrast injection for $\mathrm{CT}$ acquisition prior to transcatheter aortic valve replacement: aorta assessment and screening for coronary artery disease. Acad Radiol 26:e150-e160. https://doi.org/10.1016/j.acra. 2018.06.016

25. Matsumoto S, Yamada Y, Hashimoto M et al (2016) CT imaging before transcatheter aortic valve implantation (TAVI) using variable helical pitch scanning and its diagnostic performance for coronary artery disease. Eur Radiol 27(5):1963-1970 1-8. https://doi. org/10.1007/s00330-016-4547-4

26. Pontone G, Andreini D, Bartorelli AL et al (2011) Feasibility and accuracy of a comprehensive multidetector computed tomography acquisition for patients referred for balloon-expandable transcatheter aortic valve implantation. Am Heart J 161:1106-1113. https:// doi.org/10.1016/j.ahj.2011.03.003

27. Opolski MP, Kim W-K, Liebetrau C et al (2015) Diagnostic accuracy of computed tomography angiography for the detection of coronary artery disease in patients referred for transcatheter aortic valve implantation. Clin Res Cardiol 104:471-480. https://doi.org/ 10.1007/s00392-014-0806-Z
28. Hamdan A, Wellnhofer E, Konen E et al (2015) Coronary CT angiography for the detection of coronary artery stenosis in patients referred for transcatheter aortic valve replacement. J Cardiovasc Comput Tomogr 9:31-41. https://doi.org/10.1016/j.jcct.2014.11. 008

29. Schicchi N, Fogante M, Pirani PE et al (2020) Third generation dual source CT with ultra-high pitch protocol for TAVI planning and coronary tree assessment: feasibility, image quality and diagnostic performance. Eur J Radiol 122:108749. https://doi.org/10.1016/j. ejrad.2019.108749

30. Andreini D, Pontone G, Mushtaq S et al (2014) Diagnostic accuracy of multidetector computed tomography coronary angiography in 325 consecutive patients referred for transcatheter aortic valve replacement. Am Heart J 168:332-339. https://doi.org/10.1016/j.ahj. 2014.04.022

31. Haase R, Schlattmann P, Gueret P et al (2019) Diagnosis of obstructive coronary artery disease using computed tomography angiography in patients with stable chest pain depending on clinical probability and in clinically important subgroups: meta-analysis of individual patient data. BMJ 365:11945. https://doi.org/10. 1136/bmj.11945

32. Francone M, Budde RPJ, Bremerich J et al (2020) CT and MR imaging prior to transcatheter aortic valve implantation: standardisation of scanning protocols, measurements and reporting - a consensus document by the European Society of Cardiovascular Radiology (ESCR). Eur Radiol 30:2627-2650. https://doi.org/10.1007/s00330-019-06357-8

33. Lewis MA, Pascoal A, Keevil SF, Lewis CA (2016) Selecting a CT scanner for cardiac imaging: the heart of the matter. Br J Radiol 89: 20160376. https://doi.org/10.1259/bjr.20160376

34. Goel SS, Ige M, Tuzcu EM et al (2013) Severe aortic stenosis and coronary artery disease-implications for management in the transcatheter aortic valve replacement era: a comprehensive review. J Am Coll Cardiol 62:1-10. https://doi.org/10.1016/j.jacc.2013.01. 096

35. Min JK, Shaw LJ, Devereux RB et al (2007) Prognostic value of multidetector coronary computed tomographic angiography for prediction of all-cause mortality. J Am Coll Cardiol 50:11611170. https://doi.org/10.1016/j.jacc.2007.03.067

36. Hadamitzky M, Achenbach S, Al-Mallah M et al (2013) Optimized prognostic score for coronary computed tomographic angiography: results from the CONFIRM registry (COronary CT Angiography EvaluatioN For Clinical Outcomes: An InteRnational Multicenter Registry). J Am Coll Cardiol 62:468-476. https://doi.org/10.1016/j. jacc.2013.04.064

37. Schuetz GM, Schlattmann P, Dewey M (2012) Use of $3 \times 2$ tables with an intention to diagnose approach to assess clinical performance of diagnostic tests: meta-analytical evaluation of coronary CT angiography studies. BMJ 345:e6717. https://doi.org/10.1136/ bmj.e6717

Publisher's note Springer Nature remains neutral with regard to jurisdictional claims in published maps and institutional affiliations. 